International Journal of Pure and Applied Mathematics

Volume 111 No. 2 2016, 357-360

ISSN: 1311-8080 (printed version); ISSN: 1314-3395 (on-line version)

url: http://www.ijpam.eu

doi: 10.12732 /ijpam.v111i2.18

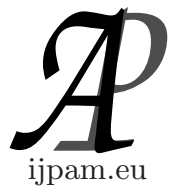

\title{
A QUESTION ON INDISCERNIBLES
}

\author{
Martin Dowd \\ 60 Mooring Ln. \\ Daly City, CA 94014, USA
}

\begin{abstract}
The question is considered, whether for some limit ordinal $\alpha, L_{\alpha}$ has an infinite set of indiscernibles. This is true if $\alpha$ is an $\omega$-Erdos cardinal. Whether the hypothesis can be weakened is a question of interest.
\end{abstract}

AMS Subject Classification: 03E55

Key Words: indiscernibles

\section{Introduction}

Let II denote the statement: for some limit ordinal $\alpha, L_{\alpha}$ has an infinite set of indiscernibles (ordinals equipped with their natural order). It is well-known that if there is an $\omega$-Erdos cardinal (a cardinal $\kappa$ such that $\kappa \rightarrow(\omega)^{<\omega}$ ) then II holds (see theorem 9.3 of [2]). In particular $\neg$ II is a very strong statement, implying that $\omega$-Erdos cardinals do not exist.

It is a question of interest whether II be deduced from a weaker hypothesis than the existence of an $\omega$-Erdos cardinal. It is also of interest what properties $\alpha$ must have for $L_{\alpha}$ to have indiscernibles.

It is also of interest whether $\mathrm{II}^{L}$ holds. Since $\alpha \mapsto L_{\alpha}$ and the satisfaction predicate are absolute, $\mathrm{II}^{L}$ holds iff there as a limit ordinal $\alpha$ and a set $I \in L$ such that $I$ is a set of indiscernibles for $L_{\alpha}$.

$\begin{array}{lr}\text { Received: } & \text { October 18, } 2016 \\ \text { Revised: } & \text { November 24, } 2016 \\ \text { Published: } & \text { December 11, } 2016\end{array}$

(c) 2016 Academic Publications, Ltd. url: www.acadpubl.eu 
Theorem 1. If $I I^{L}$ holds then II holds.

Proof. This follows by the remarks preceding the theorem.

Since theorem 9.3 of [2] holds in $L, \mathrm{II}^{L}$ holds if there is an $\omega$-Erdos cardinal in $L$, and this holds if there is an $\omega$-Erdos cardinal (theorem 9.15 of [2]),

\section{Basic Facts}

It is well-known (see [1]) that there is a collection of function definitions $\left\{h_{\phi}\right\}$ such that $h_{\phi}$ defines a Skolem function for $\phi$ in $L_{\alpha}$ for any limit ordinal $\alpha$. The function defined in $L_{\alpha}$ will be denoted $h_{\phi}^{L_{\alpha}}$, or $h_{\phi}$ if there is no danger of confusion. The Skolem hull of $S \subseteq L_{\alpha}$ will always be taken using these functions, and denoted $H(S)$.

Let $I$ be a set of indiscernibles for $L_{\alpha}$. For $S \subseteq L_{\alpha}$ the transitive collapse of $H(S)$ is isomorphic to $L_{\tilde{\alpha}}$ for some $\tilde{\alpha}$; the composition $j: L_{\tilde{\alpha}} \mapsto L_{\alpha}$ of the isomorphism with inclusion is an elementary embedding. Consequently, $j^{-1}[S]$ is a set of indiscernibles for $L_{\tilde{\alpha}}$.

Theorem 2. If II holds then there is a countable $\alpha$ such that $L_{\alpha}$ has an infinite set of indiscernibles $I$, and such that $L_{\alpha}=H(I)$.

Proof. Let $J$ be a set of indiscernibles for $L_{\beta}$. Let $S$ be the first $\omega$ elements of $J$. Let $L_{\alpha}$ be the transitive collapse of $H(S)$. Let $I=j^{-1}[S]$.

Theorem 3. If II holds then it is not provable in ZFC that II implies the existence of inaccessible cardinals.

Proof. By theorem 2 and absoluteness, if II holds then it holds in $V_{\kappa}$ where $\kappa$ is the smallest inaccessible. If it were provable that II implied that an inaccessible cardinal existed, then an inaccessible cardinal would exist in $V_{\kappa}$, which is a contradiction.

Theorem 4. If $I I^{L}$ holds then there is an $\alpha<\omega_{1}^{L}$ such that $L_{\alpha}$ has an infinite set of indiscernibles $I \in L$, and such that $L_{\alpha}=H(I)$.

Proof. The proof of theorem 2 is an argument in ZFC. Note that by absoluteness $H(S)$ is the same in $L$ and $V$.

Theorem 5. If $I I^{L}$ holds then it is not provable in $Z F C$ that $I I^{L}$ implies the existence of inaccessible cardinals in $L$. 
Proof. As in the proof of theorem 3, if $\mathrm{II}^{L}$ holds then it holds in $L_{\kappa}$ where $\kappa$ is the smallest inaccessible in $L$.

\section{3. $F_{n}$-Indiscernibles}

Let $F$ be the class of augmented formulas in the language of set theory expanded by symbols for the Skolem functions, where an augmented formula $\phi\left(x_{1}, \ldots, x_{n}\right)$ is a formula $\phi$ together with a sequence $x_{1}, \ldots, x_{n}$ of variables, which includes the free variables of $\phi$. For $C \subseteq F$ and $\alpha$ a limit ordinal, a subset $I \subseteq \alpha$ is said to be a set of $C$-indiscernibles for $L_{\alpha}$ if for all $\phi\left(x_{1}, \ldots, x_{n}\right) \in C$, and sequences $\gamma_{1}<\cdots \gamma_{n}$ and $\delta_{1}<\cdots<\delta_{n}$ of elements of $I, \models_{L_{\alpha}} \phi\left(\gamma_{1}, \ldots, \gamma_{n}\right) \Leftrightarrow$ $\phi\left(\delta_{1}, \ldots, \delta_{n}\right) . F$-indiscernibles are called simply indiscernibles.

Let $F_{n}$ denote the formulas of $F$, where the variable sequence has length at most $n$. For a cardinal $\kappa$ and an integer $n$ let $\operatorname{IE}(\kappa, n)$ be defined by the recursion: $\operatorname{IE}(\kappa, 0)=\kappa, \operatorname{IE}(\kappa, n+1)=2^{\operatorname{IE}(\kappa, n)}$.

Theorem 6. For an integer $n>0, L_{\kappa}$ has a set of $F_{n}$-indiscernibles of order type $\left(2^{\aleph_{0}}\right)^{+}$where $\kappa=\operatorname{IE}\left(\aleph_{0}, n\right)^{+}$.

Proof. By the Erdos-Rado theorem (theorem 7.3 of [2]), $\kappa \rightarrow\left(\left(2^{\aleph_{0}}\right)^{+}\right)_{2^{\aleph_{0}}}^{n}$. As in the proof of lemma 17.24 of [1], let $F:[\kappa]^{n} \mapsto \operatorname{Pow}\left(F_{n}\right)$ be the function where $F\left(\gamma_{1}, \ldots, \gamma_{n}\right)=\left\{\phi\left(x_{1}, \ldots, x_{n}\right) \in F_{n}: \models_{L_{k}} \phi\left(\gamma_{1}, \ldots, \gamma_{n}\right)\right.$. There is a homogeneous set for this partition, and it is a set of indiscernibles as required.

\section{Atomic Formulas}

Let $A$ be the set of atomic formulas of $F$, and let $A_{n}$ be the set of atomic formulas of $F_{n}$.

Theorem 7. A set of $A$-indiscernibles for $L_{\alpha}$ is a set of $F$-indiscernibles. $A$ set of $A_{n}$-indiscernibles for $L_{\alpha}$ is a set of $F_{n}$-indiscernibles.

Proof. Let $I$ be a set of $A$-indiscernibles. By induction on the formation of $\phi, I$ is a set of indiscernibles for $\phi$. This follows by hypothesis for atomic formulas. The induction step for a propositional connective is straightforward. For $\phi=\exists y \psi(y, \vec{x})$, inductively $I$ is a set of indiscernibles for $\psi\left(h_{\psi}(\vec{x}), \vec{x}\right)$, and hence for $\phi$. 
Subsets of $A$ lead to questions of interest. In particular, let $E$ be the set of equations. It is of interest whether there is an $L_{\alpha}$ with an infinite set of $E$-indiscernibles, or whether the value of $\kappa$ in theorem 6 can be improved for $E_{n}$-indiscernibles.

Let $E_{r}$ be the equations $y=t(\vec{x})$, where in the variable sequence for this formula, $y$ can occur at any point in $\vec{x}$.

Theorem 8. $I$ is a set of $E_{r}$-indiscernibles for $L_{\alpha}$ iff every formula of $E_{r}$ has the value false at sequences from $I$. The same holds for $E_{r n}$ for $n \in \omega$.

Proof. Suppose $I$ is a set of $E_{r}$-indiscernibles. Let $x_{1}, \ldots, x_{i-1}, y, x_{i+1}, \ldots, x_{n}$ be the variable list for $y=t$. Let $\alpha_{1}<\cdots<\alpha_{n}$ be elements of $I$. It may be assumed that $\alpha_{i+1}$ in not the successor of $\alpha_{i}$ in the enumeration of $I$; let $\beta$ be the successor. If $y=t$ is true then $\beta=\alpha_{i}$, a contradiction. Hence $y=t$ is false. The converse implication is trivial.

The same questions can be asked for $E_{r}$ as for $E$. Let $E_{r l}$ be the equations of $E_{r}$, where $y$ is at the end of the variable sequence.

Theorem 9. $L_{\aleph_{1}}$ has a set of $E_{r l}$-indiscernibles of order type $\aleph_{1}$.

Proof. Define the element $i_{\beta}$ of $I$ recursively as the least element which is not in the Skolem hull of $\left\{i_{\gamma}: \gamma<\beta\right\}$.

\section{References}

[1] T. Jech, Set Theory, Springer, Germany, 2003.

[2] A. Kanamori, The Higher Infinite, Springer-Verlag, Germany, 2003. 